\title{
On two Algorithmic Problems about Synchronizing Automata
}

\author{
Mikhail V. Berlinkov^ \\ Institute of Mathematics and Computer Science, \\ Ural Federal University, 620000 Ekaterinburg, Russia \\ berlm@mail.ru
}

\begin{abstract}
Under the assumption $\mathcal{P} \neq \mathcal{N} \mathcal{P}$, we prove that two natural problems from the theory of synchronizing automata cannot be solved in polynomial time. The first problem is to decide whether a given reachable partial automaton is synchronizing. The second one is, given an $n$-state binary complete synchronizing automaton, to compute its reset threshold within performance ratio less than $d \ln (n)$ for a specific constant $d>0$.
\end{abstract}

\section{Testing for synchronization}

A deterministic finite automata (DFA) $\mathscr{A}$ is a triple $\langle Q, \Sigma, \delta\rangle$ where $Q$ is the state set, $\Sigma$ is the input alphabet and $\delta: Q \times \Sigma \rightarrow Q$ is the transition function. If $\delta$ is totally defined on $Q \times \Sigma$ then $\mathscr{A}$ is called complete, otherwise $\mathscr{A}$ is called partial.

The transition function can be naturally extended to $\Sigma^{*}$ as follows. For every state $q \in Q$ and $u \in \Sigma^{*}$ we let $\delta(q, \lambda)=q$ where $\lambda$ is an empty word, and we inductively define $\delta(q, u a)=\delta(\delta(q, u), a)$ for any $a \in \Sigma$ provided that $\delta(q, u)$ and $\delta(\delta(q, u), a)$ are defined. We can simplify the notation by writing $S . w$ instead of $\{\delta(q, w) \mid q \in S\}$ for a subset $S \subseteq Q$ and a word $w \in \Sigma^{*}$.

A DFA $\mathscr{A}=\langle Q, \Sigma, \delta\rangle$ is called synchronizing if there exists a word $w \in \Sigma^{*}$ such that $|Q . w|=1$. Notice that here, in contrast to some other versions of synchronizability studied in the realm of partial automata (see e.g. [8]), $w$ is not assumed to be defined at all states. Each word $w$ with this property is said to be a reset word for $\mathscr{A}$. The minimum length of reset word is called the reset threshold of $\mathscr{A}$ and denoted by $r t(\mathscr{A})$. Analogously, a word $w$ synchronizes a subset $S \subseteq Q$ if $|S . w|=1$.

Recall that a DFA $\mathscr{A}=\langle Q, \Sigma, \delta\rangle$ is called reachable if one can choose an initial state $q_{0} \in Q$ and a final set of states $F \subseteq Q$ such that each state

\footnotetext{
* Supported by the Presidential Programme for young researchers, grant MK3160.2014.1 and by the Russian Foundation for Basic research, grant 13-01-00852
} 
$q \in Q$ is accessible from $q_{0}$ and co-accessible from $F$, i.e. there are words $u, v \in \Sigma^{*}$ such that $q_{0} . u=q$ and $q . v \in F$. This case is of certain interest due to its applications in dna-computing, namely, reset words for partial reachable automata serve as constants for the corresponding splicing systems (see e.g. [3]). It is known that the problem of testing whether or not a given strongly connected partial automaton is synchronizing can be solved in polynomial time (see [12, Algorithm 3]). In contrast, we show here that the problem becomes PSPACE-complete if we allow automata to be reachable instead of being strongly-connected.

Now we adapt the following results from [13] about subset synchronization in complete strongly connected automata to our case.

Theorem 1 ([13, Theorem 7]). There is a series of strongly connected binary automata $\mathscr{A}_{n}=\left\langle Q_{n},\{a, b\}, \delta_{n}\right\rangle$ and corresponding subsets $S_{n} \subset Q_{n}$ such that the minimum length of synchronizing words for $S_{n}$ in $\mathscr{A}_{n}$ has $\operatorname{order} 2^{\Omega(n)}$.

Theorem 2 ([13, Theorem 10]). Given a strongly connected binary automaton $\mathscr{A}=\langle Q,\{a, b\}, \delta\rangle$ and a subset of states $S \subset Q$, it is PSPACEcomplete to decide whether or not $S$ can be synchronized in $\mathscr{A}$.

Let us present a transparent reduction to the problem of synchronization of partial automata in the following lemma.

Lemma 1. For each complete strongly connected binary automaton $\mathscr{A}=$ $\langle Q,\{a, b\}, \delta\rangle$ and a subset $S \subseteq Q$ one can construct in $O(|Q|)$ time a reachable partial 3-letter automaton $\mathscr{B}=\left\langle Q^{\prime},\{a, b, c\}, \delta^{\prime}\right\rangle$ with at most $2|Q|$ states such that:

1. If $u \in\{a, b\}^{*}$ synchronizes $S$ in $\mathscr{A}$ then the word cu synchronizes $\mathscr{B}$;

2. If $w \in\{a, b, c\}^{*}$ synchronizes $\mathscr{B}$ then $w$ has a suffix $u \in\{a, b\}^{*}$ such that $u$ synchronizes $S$ in $\mathscr{A}$;

3. $S$ can be synchronized in $\mathscr{A}$ if and only if $\mathscr{B}$ is synchronizing;

4. If $S$ can not be synchronized in $\mathscr{A}$ by words of length less than $R$ then the reset threshold of $\mathscr{B}$ is at least $R$.

Proof. Denote $k=|S|$ and let $S=\left\{s_{0}, s_{1}, \ldots, s_{k-1}\right\}$ and $Q^{\prime}=Q \cup Z$ where $Z=\left\{z_{0}, z_{1}, \ldots, z_{k-1}\right\}$ is the set of new states.

Define the transition function $\delta^{\prime}$ as follows.

$$
\delta^{\prime}(q, x)= \begin{cases}\delta(q, x), & q \in Q, x \in\{a, b\} ; \\ s_{i}, & q=z_{i}, x=c ; \\ z_{(i+1 \quad \bmod k)}, & q=z_{i}, x \in\{a, b\} .\end{cases}
$$


Since $\mathscr{A}$ is strongly connected and there is the cycle by $Z, \mathscr{B}$ is reachable for $q_{0}=z_{0}$ and $F=Q$.

Since $Q^{\prime} . c=S \subseteq Q$ while $c$ is undefined on $Q$ and the action of the letters $a, b$ coincides in $\mathscr{A}$ and $\mathscr{B}$ on $Q$, Claim 1 follows. Since $w$ synchronizes $\mathscr{B}$ while $Z . a=Z . b=Z$ and $c$ is undefined on the states from $Q$, we conclude that $w$ should be of the form $v c u$ where $u, v \in\{a, b\}^{*}$. Since $Q^{\prime} \cdot v c=S$, the word $u$ should synchronize $S$ and Claim 2 follows. Claims 3 and 4 immediately follow from Claims 1 and 2 .

Theorem 3. Testing a given reachable partial 3-letter automaton for synchronization is PSPACE-complete. There is a series of reachable partial 3-letter $n$-state automata with reset thresholds of order $2^{\Omega(n)}$.

Proof. The problem is PSPACE-hard by Theorem 2 and Claim 3 of Lemma 1. The corresponding series of reachable automata exists by Theorem 1 and Claim 4 of Lemma 1 .

It remains to prove that the problem belongs to $P S P A C E$. Given a reachable partial automaton $\mathscr{A}=\langle Q, \Sigma, \delta\rangle$ we store only a current subset $S$ initialized by $Q$. In an endless loop, we nondeterministically choose a letter $a \in \Sigma$ and let $S:=S . a$. If at some step $|S|=1$ we return "yes", otherwise continue the iteration. Since for this algorithm $O(n)$ memory is enough, we have an NPSPACE algorithm which is PSPACE by the Savitch's theorem [10].

The following lemma relies on the usual technique of encoding letters in states (see e.g. [2]).

Lemma 2. For each reachable d-letter partial automaton $\mathscr{A}=\langle Q, \Sigma, \delta\rangle$, one can construct in polynomial time a binary reachable partial automaton $\mathscr{B}$ such that $\mathscr{A}$ is synchronizing if and only if $\mathscr{B}$ is synchronizing, $\left|Q^{\prime}\right|=$ $d|Q|$ and $r t(\mathscr{A}) \leq r t(\mathscr{B}) \leq d * r t(\mathscr{A})+1$ if $\mathscr{A}$ is synchronizing.

Proof. Let $\Sigma=\left\{a_{1}, a_{2}, \ldots, a_{d}\right\}$ we construct $\mathscr{B}=\left\langle Q^{\prime},\{a, b\}, \delta^{\prime}\right\rangle$ as follows. We let $Q^{\prime}=Q \times \Sigma$ and define the transition function $\delta^{\prime}: Q^{\prime} \times$ $\{a, b\} \rightarrow Q^{\prime}$ as follows:

$$
\delta^{\prime}\left(\left(q, a_{i}\right), x\right)= \begin{cases}\left(q, a_{\min (i+1, d)}\right) & \text { if } x=a, \\ \left(\delta\left(q, a_{i}\right), a_{1}\right) & \text { if } x=b \text { and } a_{i} \text { is defined on } q \\ \text { undefined otherwise. } & \end{cases}
$$

Thus, the action of $a$ on a state $q^{\prime} \in Q^{\prime}$ substitutes an appropriate letter from the alphabet $\Sigma$ of $\mathscr{A}$ for the second component of $q^{\prime}$ while the 
action of $b$ imitates the action of the second component of $q^{\prime}$ on its first component and resets the second component to $a_{1}$.

Given a word $w=a^{i_{1}} b a^{i_{2}} b \ldots a^{i_{k}} b \in\{a, b\}^{*}$, let $r(w)$ be a reduced word $a^{\min \left(i_{1}, d\right)} b a^{\min \left(i_{2}, d\right)} b \ldots a^{\min \left(i_{k}, d\right)} b$ in $\{a, b\}^{*}$. Besides that, we define the map $f:\{a, b\}^{*} \mapsto \Sigma^{*}$ by $f(w)=a_{\min \left(i_{1}, d\right)} a_{\min \left(i_{2}, d\right)} \ldots a_{\min \left(i_{k}, d\right)}$. Given $w \in\{a, b\}^{*}$, by the definition of $f$ we get that if $f(w)$ resets $\mathscr{A}$ then $b w$ resets $\mathscr{B}$, and if $w$ resets $\mathscr{B}$ then $f^{-1}(r(w))$ resets $\mathscr{A}$. The lemma follows.

As a straightforward corollary of Theorem 3 and Lemma 2 (for $d=3$ ) we get the main result of this section.

Corollary 1. Testing a given reachable partial binary automaton for synchronization is PSPACE-complete. There is a series of reachable partial binary $n$-state automata with reset thresholds of order $2^{\Omega(n)}$.

\section{Approximation of reset thresholds}

In this section we restrict ourself to the case of complete automata. For this case, testing for synchronization is polynomial. When an automaton is synchronizing, the next natural problem is to calculate its reset threshold. It is known that a precise calculation of the reset threshold is computationally hard (see e.g. [4, [9]). There are some polynomial time algorithms that, given a synchronizing automaton, find a reset word for it, see, e.g. [4]. These algorithms can be used as approximation algorithms for calculating the reset threshold, and it is quite natural to ask how good such a polynomial approximation can be. The quality of an approximation algorithm is measured by its performance ratio, which for our problem can be defined as follows. Let $K$ be a class of synchronizing automata. We say that an algorithm $M$ approximates the reset threshold in $K$ if, for an arbitrary DFA $\mathscr{A} \in K$, the algorithm calculates an integer $M(\mathscr{A})$ such that $M(\mathscr{A}) \geq r t(\mathscr{A})$. The performance ratio of $M$ at $\mathscr{A}$ is $R_{M}(\mathscr{A})=\frac{M(\mathscr{A})}{r t(\mathscr{A})}$. The author [2] proved that, unless $\mathcal{P}=\mathcal{N} \mathcal{P}$, for no constant $r$, a polynomial time algorithm can approximate the reset threshold in the class of all binary synchronizing automata with performance ratio less than $r$.

When no polynomial time approximation within a constant factor is possible, the next natural question is whether or not one can approximate within a logarithmic factor. Gerbush and Heeringa [5] conjectured that if $\mathcal{P} \neq \mathcal{N} \mathcal{P}$, then there exists $\alpha>0$ such that no polynomial time algorithm approximating the reset threshold in the class of all synchronizing automata with a fixed number $k>1$ of input letters achieves the 
performance ratio $\alpha \log |Q|$ at all DFAs $\langle Q, \Sigma, \delta\rangle$. Using a reduction from the problem Set-Cover and a powerful non-approximation result from [1, Gerbush and Heeringa proved a weaker form of this conjecture when the number of input letters is allowed to grow with the state number.

Here we prove the conjecture from [5] in its full generality, for each fixed size $k>1$ of the input alphabet. Though we depart from the same reduction from Set-Cover as in [5], we use not only the result from [1, but also some ingredients from its proof, along with an encoding of letters in states.

Let us follow [1] and [5] below. Given a universe $\mathcal{U}=\left\{u_{1}, \ldots, u_{n}\right\}$ and a family of its subsets, $\mathcal{S}=\left\{S_{1}, \ldots, S_{m}\right\} \subseteq P(\mathcal{U})$ such that $\bigcup_{S_{j} \in \mathcal{S}} S_{j}=\mathcal{U}$, Set-Cover is the problem of finding there a minimal sub-family $C \subseteq \mathcal{S}$ that covers the whole universe in the sense that $\bigcup_{S_{j} \in \mathrm{C}} S_{j}=\mathcal{U}$. Denote the size of the minimal sub-family by $\operatorname{OPT}(\mathcal{U}, \mathcal{S})$. Set-Cover is a classic $\mathcal{N P}$-hard combinatorial optimization problem, and it is known that it can be approximated in polynomial time to within $\ln (n)-\ln (\ln (n))+\Theta(1)$ (see [7/1]).

The following transparent reduction from Set-Cover is presented in [5]. Given a Set-Cover instance $(\mathcal{U}, \mathcal{S})$, define the automaton

$$
\mathscr{A}(\mathcal{U}, \mathcal{S})=\left\langle\mathcal{U} \cup\{\hat{q}\}, \Sigma=\left\{a_{1}, \ldots a_{m}\right\}\right\rangle
$$

where the transition function is defined as follows.

$$
\delta\left(u, a_{i}\right)= \begin{cases}\hat{q}, & u \in S_{i} \\ u, & u \notin S_{i} .\end{cases}
$$

Remark 1. Let $\mathscr{A}=\langle Q, \Sigma, \delta\rangle$ be the automaton defined by $(\mathcal{U}, \mathcal{S})$ as above. Then $\operatorname{rt}(\mathscr{A})=\operatorname{OPT}(\mathcal{U}, \mathcal{S}), \quad|Q|=|\mathcal{U}|+1, \quad|\Sigma|=|\mathcal{S}|$.

The following powerful result has been obtained in [1].

Theorem 4 ([1, Theorem 7]). Unless $\mathcal{P}=\mathcal{N} \mathcal{P}$, no polynomial time algorithm can approximate Set-Cover within performance ratio less than $c_{s c} \ln n$ where $n$ is the size of the universe and $c_{s c}>0.2267$ is a specific constant.

Here we prove the aforementioned conjecture from [5] by encoding binary representation of letters in states and using some properties from the proof of Theorem 4 . 
Lemma 3. For every $m$-letter synchronizing automaton $\mathscr{A}=\langle Q, \Sigma, \delta\rangle$, there is a 2-letter synchronizing automaton $\mathscr{B}=\mathscr{B}(\mathscr{A})=\left\langle Q^{\prime},\{0,1\}, \delta^{\prime}\right\rangle$ such that

$$
r t(\mathscr{A})\left\lceil\log _{2} m+1\right\rceil \leq r t(\mathscr{B}) \leq\left\lceil\log _{2} m+1\right\rceil(1+r t(\mathscr{A})),
$$

$\mathscr{B}$ has at most $4 m|Q|$ states and can be constructed in polynomial time of $m$ and $|Q|$.

Proof. Let $\Sigma=\left\{a_{1}, \ldots, a_{m}\right\}$ and for simplicity assume that $m$ is a power of 2, i.e. $m=2^{k}$ (otherwise we can add at most $m-1$ letters with trivial action without impact on the bounds). Let $\ell:\{0,1\}^{k} \mapsto \Sigma$ be a bijective function. Set $Q^{\prime}=Q \times\{0,1\} \leq k$ and define the transition function $\delta^{\prime}: Q^{\prime} \times\{0,1\} \rightarrow Q^{\prime}$ as follows. For each $q \in Q$, each binary sequence $w \in\{0,1\}^{\leq k}$ and each bit $x \in\{0,1\}$, we let

$$
\delta^{\prime}((q, w), x)= \begin{cases}(q, w x) & \text { if }|w|<k \\ (q \cdot \ell(w), \lambda) & \text { if }|w|=k, x=1 \\ (q, w) & \text { if }|w|=k, x=0\end{cases}
$$

Let $u=a_{j_{1}} a_{j_{2}} \ldots a_{j_{t}}$ be a reset word for $\mathscr{A}$. Then the word

$$
1^{k+1} \ell^{-1}\left(a_{j_{1}}\right) 1 \ldots \ell^{-1}\left(a_{j_{t}}\right) 1
$$

is reset for $\mathscr{B}$ and its length equals $(k+1)(t+1)$. The upper bound follows.

In order to prove the lower bound it is enough to consider the shortest binary word $u$ which synchronizes the subset $(Q, \lambda)$ in $\mathscr{B}$. Since $u$ is chosen shortest, $u=w_{1} 1 w_{2} 1 \ldots w_{r} 1$ where $\left|w_{j}\right|=k$ for each $j \in\{1, \ldots r\}$. Indeed, after applying a word $w \in\{0,1\}^{k}$ to the state of the form $(q, \lambda)$ it make no sense to apply 0 in view of the third choice of definition 4 . Then the word $\ell\left(w_{1}\right) \ell\left(w_{2}\right) \ldots \ell\left(w_{r}\right)$ resets $\mathscr{A}$ and the lower bound follows.

Now, suppose that for some constant $d>0$, there is a polynomial time algorithm $f_{2}$ such that

$$
r t(\mathscr{B}) \leq f_{2}(\mathscr{B}) \leq d \ln (n) r t(\mathscr{B})
$$

for every 2-letter $n$-state synchronizing automaton $\mathscr{B}$. Then Lemma 3 implies that for each $m \geq 2$ there is also a polynomial time algorithm $f_{m}$ such that

$$
r t(\mathscr{A}) \leq f_{m}(\mathscr{A}) \leq d \ln (4 n m)(1+r t(\mathscr{A}))
$$


for every $m$-letter $n$-state synchronizing automaton $\mathscr{A}$. Indeed, such algorithm first constructs $\mathscr{B}(\mathscr{A})$ with at most $4 n m$ states as in Lemma 3 , and then runs $f_{2}$ on $\mathscr{B}(\mathscr{A})$ :

$$
r t(\mathscr{A}) \leq f_{m}(\mathscr{A})=\frac{f_{2}(\mathscr{B}(\mathscr{A}))}{\left\lceil\log _{2} m+1\right\rceil} \leq d \ln (4 n m)(r t(\mathscr{A})+1) .
$$

Combining this with Theorem 4 and Remark 1 we immediately get the following corollary.

Corollary 2. Let $g(n)$ be an upper bound on the cardinality of the set family $\mathcal{S}$ as a function of the size of the universe $\mathcal{U}$ from the reduction to Set-Cover from [1]. Then, unless $\mathcal{P}=\mathcal{N} \mathcal{P}$, no polynomial time algorithm approximates reset threshold within performance ratio $\frac{d}{\log _{n} g(n)+1} \ln (n)$ for any $d<c_{s c}$ in the class of all 2-letter synchronizing automata.

Thus it suffices to find a lower bound on the size of the universe $\mathcal{U}$ and an upper bound on the size of the family of subsets $\mathcal{S}$ in the reduction to Set-Cover presented in [1]. Namely, we need to find a polynomial upper bound for $g(n)$.

Due to the space limit, we shall use some notation from [1] without reproducing all definitions. First, the universe $\mathcal{U}$ is defined as $[D] \times \Phi \times B$ where $D=\left\lfloor\frac{|\Phi|}{\eta|X|}\right\rfloor, \eta$ is a constant. Hence the rough lower bound for the size of the universe $\mathcal{U}$ is $|\Phi|$.

The size of the family of subsets $\mathcal{S}$ is equal to $D|X||F|+|\Phi||F|^{d}$ where $F$ is a field of cardinality at most $2^{\log _{2}^{1-\beta}|X|} \leq|X|$ and $d \geq 2$ is a positive integer which can be taken equal 3. Hence the upper bound for $|\mathcal{S}|$ is $\Theta(1)|\Phi||X|^{d}$. Thus we get that

$$
\log _{|\mathcal{U}|}|\mathcal{S}| \leq \frac{d+\log _{|X|}|\Phi|}{\log _{|X|}|\Phi|}
$$

Note that $|\Phi|$ is only restricted to be some polynomial of $|X|$, i.e. it can be chosen to be $|X|^{r}$ for an arbitrary large constant $r$. As a conclusion we get the following lemma, which gives a nice property of Set-Cover itself.

Lemma 4. Given any $\gamma>0$, unless $\mathcal{P}=\mathcal{N} \mathcal{P}$, no polynomial time algorithm approximates the Set-Cover with performance ratio $d \ln n$ for any $d<c_{s c}$ in the class of all Set-Cover instances $(\mathcal{U}, \mathcal{S})$ satisfying $\log _{|\mathcal{U}|}|\mathcal{S}| \leq 1+\gamma$

Combining this with Corollary 2 gives us the second main result. 
Theorem 5. Unless $\mathcal{P}=\mathcal{N} \mathcal{P}$, no polynomial time algorithm approximates the reset threshold within performance ratio less than $0.5 c_{s c} \ln n$ in the class of all $n$-state synchronizing automata with 2 input letters.

Let us notice that the same bound holds true for any fixed nonsingleton alphabet. Theorem [5 improves the previous result of the author [2] about non-approximability within any constant factor and also gives the positive answer to the corresponding conjecture from [5].

It is known (see e.g. [7, [11]) that the greedy algorithm for Set-Cover has a logarithmic performance ratio. Despite of relations with the problem of computing the reset threshold, there is a series of automata for which the greedy algorithm $=$ computes reset threshold with linear performance ratio ([Ananichev, 2014], unpublished). Hence the first natural open question is about the tightness of the bound in Theorem 5 .

Acknowledgements. The author thanks the anonymous referees for their useful remarks and suggestions.

\section{References}

1. Alon, N., Moshkovitz, D., Safra, S.: Algorithmic Construction of Sets for krestrictions. ACM Trans. Algorithms, 2(2), pp. 153-177 (2006)

2. Berlinkov, M.: Approximating the Minimum Length of Synchronizing Words Is Hard. Theory Comput. Syst. 54(2), pp. 211-223 (2014)

3. Bonizzoni, P., Jonoska, N: Regular Splicing Languages Must Have a Constant. In: Mauri, Giancarlo and Leporati, Alberto (eds) Developments in Language Theory, Lect. Notes Comp. Sci., 6795, pp. 82-92, Springer Berlin Heidelberg (2011)

4. Eppstein, D.: Reset Sequences for Monotonic Automata. SIAM J. Comput. 19, pp. 500-510, (1990)

5. Gerbush, M., Heeringa, B.: Approximating Minimum Reset Sequences. 15-th Implementation and application of automata, Lect. Notes Comp. Sci. 6482, pp. 154-162, Springer, Berlin (2011)

6. Ivan, S: Synchronizing Weighted Automata, arXiv:1403.5729 (2014)

7. Lovász, L.: On the Ratio of Optimal Integral and Fractional Covers. Discrete Mathematics, 13: pp. 383-390 (1975)

8. Martugin, P: Complexity of Problems Concerning Carefully Synchronizing Words for PFA and Directing Words for NFA, Lect. Notes Comp. Sci., 6072, pp. 288-302 (2010)

9. Olschewski, J., Ummels, M.: The Complexity of Finding Reset Words in Finite Automata. Lect. Notes Comp. Sci. 6281, pp. 568-579 (2010)

10. Savitch, W: Relationships Between Nondeterministic and Deterministic Tape Complexities, Journal of Computer and System Sciences 4 (2), pp. 177-192 (1970)

11. Slavik, P.: A Tight Analysis of the Greedy Algorithm for Set Cover. In Proc. 28th ACM Symp. on Theory of Computing, pp. 435-441 (1996)

12. Travers, N., Crutchfield, J.: Exact Synchronization for Finite-State Sources, J. Stat. Phys. 145:5, pp. 1181-1201 (2011)

13. Vojtěch, V: Subset Synchronization of Transitive Automata, arXiv:1403.3972 (accepted to AFL 2014). 